\title{
AUGUSTUS IN DER HIRTENWELT
}

\section{DIE DARSTELLUNG DES IDEALEN HERRSCHERS IN DER NEULATEINISCHEN BUKOLIK*}

\begin{abstract}
Summary: In the early modern age, pastoral poetry became a current genre of the praise of rulers, kings and emperors. In spite of its overwhelming richness and contemporaneous importance, this branch of the bucolic genre has received relatively little attention from researchers. Even in comprehensive works on the history of the genre, one often finds hasty remarks, e.g. that these panegyric poems were foremost influenced by Vergil's Eclogue 4.

The present paper offers a short overview of the immense diversity of the genre, paying great attention not only to Vergil, but also to the decisive influence of Calpurnius Siculus, Sannazaro and Baptista Mantuanus, as well as to the techniques of the Kreuzung der Gattungen, mainly to the interaction between pastoral and epic poetry. The analysis shows that, in spite of the huge variety of forms and the large distances in time and space, the image of the ideal emperor is surprisingly constant, and that the picture of the mythical Golden Age is almost completely drawn with the motives of the idealized reign of Augustus taken from epic poetry.
\end{abstract}

Key words: Neo-Latin literature, Renaissance Humanism, reception of antiquity, pastoral poetry, epic poetry, Augustus, eclogues, panegyric poetry, court poetry, myth of the Golden Age, image of the emperor, Vergil, Bucolics, Aeneid, Calpurnius Siculus, Petrarch, Enea Silvio Piccolomini, Naldo Naldi, Jacopo Sannazaro, Baptista Mantuanus, Lotichius Secundus, Johannes Stigelius, Nicola Giannettasio, George Buchanan

Ubi ubi es, o Auguste [...], umquam erit tempus, quo mihi liceat tua facta describere? - Servius fügt diese Bemerkung zum berühmten Proömium der achten Ekloge Vergils hinzu, ${ }^{1}$ und diese prägnante Paraphrase stellt sozusagen den Inbegriff des Bildes dar, wie der ideale Herrscher in der neulateinischen Bukolik erscheint. Dieses

* Während der Arbeiten am Aufsatz wurde der Verfasser durch das János-Bolyai-Forschungsstipendium der Ungarischen Akademie der Wissenschaften gefördert.

${ }^{1}$ 6. TV MIHI SEV MAGNI S. I. S. T. ubi ubi es, o Auguste, sive Venetiae fluenta transcendis - nam Timavus fluvius est Venetiae vel Histriae -, sive per Illyricum navigas mare, id est per Dalmatiam, putas, umquam erit tempus, quo mihi liceat tua facta describere? Servii Grammatici qui feruntur in Vergilii carmina commentarii. Recensuerunt G. THILO - H. HAGEN. Hildesheim-Zürich-New York 1986, Vol. III. 93. 
Bild wird nämlich sehr oft durch Motive der Abwesenheit, der räumlichen und zeitlichen Getrenntheit, der nostalgischen Erinnerung und des hoffnungsvollen Sehnens bestimmt. $^{2}$

Ein frühes Beispiel dafür ist die einzige Ekloge des Enea Silvio Piccolomini (1405-1464), die einige Jahrzehnte vor dem Aufblühen der Hirtendichtung, gegen 1432 geschrieben wurde ${ }^{3}$ und die Grundsituation der ersten Ekloge Vergils in ihr Gegenteil verkehrte. Am Ufer des malerischen Ortasees trifft Silvio seinen lange nicht gesehenen Freund Vegius, d.h. Maffeo Vegio, der gedrängt und verbittert in dem von wilden Tieren wimmelnden Wald herumirrt und sogar die Nächte dort verbringt, jedoch nicht wegen Liebeskummer, sondern weil er nach den Spuren des Herrschers sucht, da er vernommen hat, dass er in dieser Gegend Bären zu jagen pflegt:

nanque ego per siluas et per deserta ferarum

errabundus agor; nostri si forsitan ulla

cesaris occurrant uestigia, quando secutum

his illum accepi rigidas in montibus ursas.

$\mathrm{Zu}$ seinem Glück trifft er auf Silvius, denn von ihm erfährt er, dass seine Suche vergeblich ist - Caesar geht in dieser Gegend nie auf die Jagd. Wie Tityrus bei Vergil den heimlos gewordenen Meliboeus, so nimmt auch Silvius den müden Wanderer in Gastfreundschaft auf, denn die Nacht nähert sich (omnia villarum superat iam culmina fumus, 37). Im Gegensatz zu Meliboeus kann jedoch Vegius hoffnungsvoll in die $\mathrm{Zu}-$ kunft blicken, denn Silvius zeigt ihm den Weg und er wird zu dem als literaturliebender Mäzen bekannten Herzog von Mailand, Filippo Maria Visconti, vorgelassen. Die schmerzhafte Bemerkung des Meliboeus - fortunate senex, ergo tua rura manebunt (46) - wird von Piccolomini im Zeichen der Kontrastimitation neu formuliert, wodurch die melancholische Geschichte einen positiven Ausklang erhält:

fortunate puer, tum Cesaris ora videbis.

Bereits in diesem frühen Gedicht sind zwei charakteristische Vorgehensweisen der panegyrischen Eklogen zu beobachten. Das Umherirren des Vegius und der Lob des duca dienen lediglich als Rahmengeschichten für die in den Mittelpunkt der Ekloge gestellte, lange Erzählung (89-145), in der Silvius die Geschichte des Heiligen Julius von Novara darlegt, der noch zu Zeiten des Kaisers Theodosius in diese Gegend kam. Er befahl den Mengen von Schlangen, die die Insel bis dann unbewohnbar gemacht hatten, sie zu verlassen und baute an Stelle des Schlangennests eine Kirche. Diese

${ }^{2}$ Die Beobachtung von W. J. Kennedy über die Eklogen des Sannazaro gilt also, wie zu zeigen sein wird, auch für die panegyrischen Eklogen im Allgemeinen: „Unlike contemporary eclogues by Pontano, Castiglione, Vida and Navagero that established the mode as the premier vehicle for dirge, Sannazaro's eclogues treat the motifs of presence and absence, hope and despair, memory and desire in rich and satisfactory way.“ KENNEDY, W. S.: Sannazaro and the Uses of Pastoral. Hannover-London 1983, 179.

${ }^{3}$ Zur Datierung der Ekloge siehe PRETE, S.: Pius II on Lake Orta. In Studies in Latin Poets of the Quattrocento [University of Kansas Humanistic Studies 49]. Lawrence, University of Kansas Publications 1978, 25-49.

${ }^{4}$ Enee Silvii Picolominei postea Pii PP. II Carmina. Edidit commentarioque instruxit ADRIANUS VAN HECK. Città del Vaticano 1994. 
aitiologische Erzählung, die in der stillen Ruhe des idyllischen Abends dargeboten wird, hebt die Ekloge weit über die panegyrischen Gelegenheitsgedichte. Andererseits geht die jeglicher Individualisierung entbehrende Figur von Visconti in die an Augustus erinnernde Gestalt des idealen Herrschers dermaßen ein, dass die Figur des Cesar leicht mit dem ungarischen König und späteren deutsch-römischen Kaiser Sigismund identifiziert werden konnte. ${ }^{5}$

Einer der charakteristischsten Züge des in der Hirtendichtung dargestellten Herrscherbildes besteht vielleicht gerade in dieser Unpersönlichkeit, in der Assimilation der Figur des Herrschers an die mythischen und historischen Vorbilder. Dieses Verfahren lässt sich bereits in der ersten Ekloge des Calpurnius Siculus beobachten, die zur Zeit des Humanismus von Boiardo bis Ronsard unzähligen Dichtern als Muster diente und sogar die Wirkung der vierten Ekloge Vergils übertraf. ${ }^{6}$ In dieser Ekloge stoßen die Hirten auf eine in eine Buche geritzte, von Faunus selbst verfasste Prophezeiung, die das unter Neros Herrschaft sich anbahnende goldene Zeitalter beschreibt. Allerdings soll betont werden, dass die goldene Zeit bei Calpurnius, im Gegensatz zu Vergils vierten Ekloge, nicht mehr den Eintritt in ein sich nach kosmischen Gesetzen richtendes Weltzeitalter bedeutet, sondern bloß die Sicherheit und Friede gewährende Herrschaft des neuen Prinzeps. Anstelle der mythischen Urzeit übernimmt also die Herrschaft des Augustus die Rolle der vergangenen goldenen Zeit. Diese Betrachtungsweise war offenbar besser für den Lob von Herrschern adaptierbar als die vierte Ekloge Vergils. Es fällt auf, dass Faunus nach den einleitenden Versen der Prophezeiung der Ekloge (iuvenemque beata sequuntur / saecula, 44-45) nur die Wirkungen der Herrschaft des jungen Prinzeps schildert. Nero erscheint lediglich in den letzten fünf Zeilen. Auch dann wird sein Bild ziemlich unpersönlich nachgezeichnet: Seine Figur verschmilzt in die mythologischen und historischen Vorbilder und wird erst durch das Gedenken an Hercules, Numa Pompilius und vor allem Augustus angedeutet, im Einklang mit der zeitgenössischen Propaganda, nach der Nero der letzte Nachkomme von Aeneas und Augustus sei, ${ }^{7}$ und ex Augusti praescripto herrschen wollte. $^{8}$

In Piccolominis Ekloge verkünden nicht nur die Buchen, sondern auch die Eichen, Tannen, Haselnussbäume und Zypressen die Taten von Visconti (cesaris acta, 160): Wie er die Florentiner und die Venezianer terraque marique (169) besiegt hat und wie er als divus herrschen und das glückliche Zeitalter des Saturn wiederherstellen wird, was zugleich auch die Karriere von Vegio als Hofdichter unbedingt zu sichern scheint:

\footnotetext{
${ }^{5}$ Cesar wurde vom ersten Herausgeber der Ekloge, G. CUGNONI, mit Sigismund identifiziert: Aeneae Silvii Piccolomini Senensis qui postea fuit Pius II Pont. Max. opera inedita. Descripsit ex codicibus Chisianis vulgavit notisque illustravit JosEPHUS CUGNONI. Atti della R. Accademia dei Lincei 280 (18821883) 664-666.

${ }^{6}$ Über das Fortleben des Calpurnius siehe unseren früheren Aufsatz: SIMON, L. Z.: „,Sacra Calpurni vestigia." Calpurnio Siculo e i nuovi percorsi della bucolica umanistica nel secondo Quattrocento. StudUmanistPicen 27 (2007) 157-176.

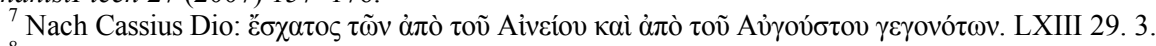

${ }^{8}$ Suetonius, Nero 10.1 .
} 
Regnabit divus confectis ordine bellis cesar et aurato revirebunt omnia seclo; nanque redit mundo Saturni certior heres. O nobis liceat tunc omnia scribere facta cesaris et magnis implere theatra coturnis. Carmina divini faciemus proxima Phebi versibus atque Linum superantia et Orphea magnum. (180-186)

Die Abwesenheit des Herrschers ist auch das bestimmende Motiv der vierten Fischerekloge des Jacopo Sannazaro (1457-1530), Proteus: Das den in Spanien gefangen gehaltenen Thronfolger, Ferdinand von Aragonien, ansprechende Proömium folgt auch hier dem Muster der achten Ekloge Vergils: Ob er von den in die Wolken ragenden Pyrenäen oder dem Ebro zurückgehalten wird, soll er die Heimkehr nicht länger aufschieben:

Tu vero, patriae Juvenis decus, edite caelo, spes generis tanti: seu te nimbosa Pyrene pro dulci Latio, pro nostris detinet arvis, seu vagus objecto munimine claudit Iberus: rumpe moras, nec te latis Hispania regnis alliciat, stirpisve tuae primordia, et ille gentis honos: licet effuso Tagus impleat auro, et Pater Oceanus spumanti perluat unda.

Nam mihi, nam tempus veniet, quum reddita sceptra Parthenopes, fractosque tua sub cuspide reges ipse canam.

Diese ist nämlich nicht nur die Bedingung für das Aufblühen von Neapel, sondern auch für den Fortschritt des Dichters, der die Musen als Erster aus den Wäldern zu den salzigen Schäumen des Meeres geführt hat (salsas deduxi primus ad undas, 19), zur epischen Dichtung: Dann wird er Ferdinands Siege selber besingen: fractosque tua sub cuspide reges / ipse canam. Der panegyrische Rahmen ist allerdings auch hier nur der Rahmen des von Vergils sechsten Ekloge inspirierten Gesanges, der langen Erzählung des Proteus über die mythische Geschichte von Neapel von der Gigantomachia bis hin zu den Heldentaten der neapolitanischen Könige und dem bitteren Schicksal von Ferdinand. ${ }^{10}$

Mit dieser Fischerekloge hat Sannazaro eine neue Spielart der panegyrischen Ekloge in Mode gebracht und auf diese Variante der Gattung eine mit der ersten Eklo-

${ }^{9}$ Jacopo Sannazaro: Latin Poetry. Translated by M. C. J. PUTNAM. The I Tatti Renaissance Library. Harvard University Press 2009.

${ }^{10} \mathrm{Zu}$ den antiken Vorbildern der Ekloge siehe neulich HuBBARD, TH.: Exile from Arcadia: Sannazaro's Piscatory Eclogues. In Pastoral Palimpsests. Essays in the Reception of Theocritus and Virgil. Ed. by M. PASCHALIS. Rethymnon Classical Studies 3 (2007) 59-77. 
ge des Calpurnius vergleichbare Wirkung ausgeübt. ${ }^{11}$ Ihm folgte eine Schar von Nachahmern, unter anderen die Familie Amalteo aus Friuli, die eine ganze Reihe von Dichtern in den Dienst der Habsburgischen Herrscher gestellt hat. ${ }^{12}$ Der Erzähler in der Fischerekloge Ioanni Austrio Proteus des Cornelio Amalteo (1530-1603) ist auch Proteus, der von der Schaffung des Menschen über die Geschichte von Io und dann Orpheus, zum Sieg von Don Juan de Austria bei Lepanto gelangt, welcher lediglich mit dem des Augustus zu vergleichen ist. Als sicher gilt, dass der Sultan sich daraufhin ins Meer stürzen wird, und es ist nur eine Frage der Zeit, bis ganz Asien befreit, Indien erobert und christianisiert wird, und die Saturnia saecula zurückkehren:

Tunc Asiae ingenti perculsus clade tyrannus

Abscindet manibus crines, atque unguibus ora.

Mox irae impatiens praerupto e vertice saxi

Sese precipitem demittet in aequoris undas.

Hinc iuga servitio solvent \& Maurus \& Indus,

Fallacisque dei ritus, legesque profanas

Contemnent, genitumque colent e Virgine numen,

Tum Parce unanimes Saturnia saecula reddent,

Et Romana ferent patrias vexilla per urbes.

Et rursus terrarum urbem Pax alma reviset.

Cornelios Bruder, Gianbattista (1528-1573), ${ }^{13}$ hat seine Ekloge Doris schon über Philipp II. von Spanien geschrieben. Die aus dem Meer emporsteigende Nereid spricht den König als geradlinigen Nachkommen von Aeneas an. ${ }^{14}$ Es kann auch kein Zweifel über den Ausklang der außerordentlich langen Prophezeiung geben: Den Triumph des Kaisers nach der Eroberung von Baktrien und Indien wird, aus einer goldenen Wolke niederblickend, Venus selber beklatschen:

${ }^{11}$ Über die Wirkung der Fischereklogen von Sannazaro auf die spanische Dichtung der Renaissance siehe BÉHAR, R.: „Tu mihi”: Variaciones bucólicas sobre un ritual de dedicatoria de Virgilio a Góngora. NRFH 61 (2013) 65-98.

12 Über die Gebrüder Amalteo siehe Ellinger, G.: Die neulateinische Lyrik Deutschlands im sechszehnten Jahrhundert. Berlin-Leipzig 1929, I $301 \mathrm{ff}$, ,Amaltheorum fratrum tam mira fuit in scribendis carminibus felicitas, tam nitidus candor, tam iucunda suavitas, ut non semel viros doctissimos, cum ea legerent, dulcedine quadam delinitos pronunciasse audiverim, aut non alium fuisse Musarum cantum, aut Musas ipsas in Amaltheos Pythagoraeo veluti commento esse transfusas" - schreibt der mit der Familie verwandte Girolamo Aleandro im Vorwort zu ihren gesammelten Gedichten: Trium fratrum Amaltheorum, Hieronymi, Io. Baptistae, Corneli Carmina. Venetiis MCLXXVII. Die Texte werden aufgrund dieser Ausgabe zitiert. Über die Ekloge Proteus siehe GRANT, W. L.: Neo-Latin Literature and the Pastoral. Chapel Hill 1965, 342-343.

${ }_{13}$ Über seine Dichtung siehe GRANT (Anm. 12) $154 \mathrm{ff}$.

${ }^{14}$ Über den trojanischen Ursprung der Habsburger siehe GARBER, J.: Trojaner - Römer - Franken Deutsche. „Nationale“ Abstammungstheorien im Vorfeld der Nationalstaatsbildung. In Nation und Literatur im Europa der Frühen Neuzeit. Akten des I. Internationalen Osnabrücker Kongresses zur Kulturgeschichte der Frühen Neuzeit. Hrsg. von K. GARBER. Frühe Neuzeit 1. Tübingen 1989, 108-163. Über die Hofpropaganda Philipps des Zweiten siehe TANnER, M.: The Last Descendant of Aeneas. The Habsburgs and the Mythic Image of the Emperor. Yale University Press 1993. 
Iamque externa tuis accesserat ora triumphis

Bellipotens, Veneris pulchro sate sanguine Caesar.

O decus, o sacer heroum flos, o bona proles

Aeneadum, nunc te patrijs, nunc inserit astris

Maior honos, \& caeca levi pede nubila vincis.

Tu Maßylum arces, Libycas tu disijcis urbes,

Tu Rhodanum, Ligerimque premis cum lucida signa,

Hostilesque tubas, \& seva perhorruit arma,

Et refluum pavidae quaerebant Naiades amnem.

Etwas zurückhaltender ist Basilio Zanchi (1501-1558), der gelehrte Bibliothekar im Vatikan, der später wegen Verdacht auf Protestantismus eingekerkert wurde. ${ }^{15}$ In seiner an Papst Leo X. geschriebenen Ekloge (Leo X. Pont. Max.) steigt Tiberinus pater selbst aus dem Wasser des Flusses empor und bewundert die kolossalen Bauarbeiten und die Herrlichkeit der mit vergoldeten Statuen ausgestatteten Tempel. Die Erinnerung an Augustus ist aber auch hier entscheidend: Tiberis bezeugt selber, dass Leo nicht vom verfluchten Verlangen nach Schätzen getrieben wird, sondern lediglich die seit Langem verfallenden Tempel der Götter würdig wiederherzustellen wünscht: ${ }^{16}$

Forte pater vitreis placidum Tyberinus ab antris

Extulerat caput, \& viridantia cornua musco:

Cum subito immensas moles, \& tecta domorum

Aequari caelo, Phrygiisque innixa columnis

Templa videt late auratis fulgentia signis:

Et laetas audit voces, atque omnia ludis

Laetitiaque videt, blanda \& resonantia pace.

[...]

Non illum argenti atque auri scelerata cupido

Vexabit: sed temple diu prolapsa Deorum

Instaurans, feret insignis praeconia famae.

$$
(1-7 ; 58-60)^{17}
$$

Das Motiv der Abwesenheit des Herrschers wird auch von Johannes Stigelius (15151562), Professor der Universität zu Wittenberg, den Karl V. bereits im Alter von 26 Jahren zum Poeten gekrönt hat, auf virtuose Weise bearbeitet. Ein Jahr darauf, zur Zeit des Feldzuges des Kaisers gegen Algerien (1542), entstand Stigelius' erste Ekloge, Iolas, die wiederum das Proömium der achten Ekloge Vergils wachruft: Ob er die Fel-

${ }^{15}$ Über sein Leben siehe ELLINGER (Anm. 12) $267 \mathrm{ff}$.

${ }^{16}$ Die Restaurierung von Rom bildete bereits unter Maximilian I. Teil der deutsch-römischen kaiserlichen Propaganda: „The restoration of Roman temples by the new crusading hero can thus be seen as part of the spiritual regeneration of the city. The classical ruins which Maximilian promises to rebuild are symbolic of the Pax Romana whereby Rome as the new Jerusalem will be the centre of a new Christian Empire.“ GWYNNE, P.: 'Tu alter Caesar eris': Maximilian I, Vladislav II, Johannes Michael Nagonius and the renovatio Imperii. Renaissance Studies 10 (1996) 65.

${ }^{17}$ Basilii Zanchii Bergomatis Canonici Ordinis Lateranensis Poemata quae extant omnia. Nunc primum ex Oporiniana editione accuratissime recensita, illustrata, \& aucta. Bergomi MDCCXLVII. 
sen des hohen Pelorus überwindet, ihn am Ebro der heimatliche Abendstern sieht oder die italische Sirene festhält, von ihm erwartet er die Inspiration, denn es wird die Zeit kommen, zu der er die Taten des Kaisers besingen und in die Rinde von Bäumen ritzen wird; dessen wird aber nicht so sehr die Buche, sondern vielmehr die Zeder würdig:

Tu mihi, seu summi superas iam saxa Pelori,

Seu videt Hesperio patrius te vesper Hibero,

Itala seu laetis Siren te detinet hortis,

Seu juga (dij faciant) nostri colis ardua Rhaeni,

Aspira, tibi numen inest, tibi numen Iola,

en erit, ut tandem calamo tua dicere facta

ordiar, incisis mandans tua nomina fagis,

o digna inscribi soli tua nomina cedro.

Weil Karl jedoch in Algerien ums Leben gekommen sein soll, baut Stigelius sein Gedicht nach dem Muster der fünften Ekloge Vergils auf. Dementsprechend erhält der Kaiser die Züge des mit Iulius Caesar identifizierten Daphnis: Er lehrte, die tyrischen Tiger unter das Joch zu schicken, ille iugo docuit Tyrios subiungere Tigres, gleichzeitig besiegt er, dem Herkules ähnlich, den Sequanicus Cacus, d.h. den französischen König Franz I. Die Abwesenheit des Herrschers ist eng mit dem soterischen Charakter seiner Heimkehr verbunden. ${ }^{19}$ Folglich ruft auch die Gestalt von Karl die Figur des am Ende des ersten Buches der Georgica als Retter angesprochenen Octavian wach:

Sis bonus, ô foelixque tuis, si vivis, Iola, Aspice turbati faciem, \& discrimina ruris, Affer opem miseris, dubijs \& consule rebus.

Es ist kein Zufall, dass die Invokation am Anfang der Georgica (hunc saltem everso iuvenem succurrere saeclo ne prohibete, I 500-501) sozusagen zum Wandermotiv wird: Sie erscheint in der erwähnten, dem Papst Leo gewidmeten Ekloge des Zanchi:

Te vero pater afflictis Leo maxime rebus

Praesidium, per quem Romana potentia caelo

Attollet caput, \& famam superinseret astris,

Dii patrii indigetes, Divûmque aeterna potestas,

${ }^{18}$ Humanistische Lyrik des 16. Jahrhunderts: lateinisch und deutsch. In Zusammenarbeit mit CHRIstof BodAmer [et al.] ausgew., übers., erl. und hrsg. von W. KÜHLMANN, R. SEIDEL und H. WIEGAND. Frankfurt am Main 1997.

${ }_{19}$ „The symbolism of the empire of Charles $\mathrm{V}$, which seemed able to include the whole world as then known and to hold out the promise of a return to spiritual unity through a revival of the cementing power of the Christianized imperial virtues, was a comforting phantom in the chaotic world of the sixteenth century." YATES, F. A.: Astraea. The Imperial Theme in the Sixteenth Century. London-Boston 1975, 27. Über die Hofpropaganda Karls V. siehe auch BÉHAR, R.: „In medio mihi Caesar erit“: Charles-Quint et la poésie impériale. e-Spania. Revue interdisciplinaire d'études hispaniques médiévales et modernes. 13 juin 2012. http://e-spania.revues.org/21140. Zuletzt gesehen am 12. Juni 2015. 
Concedant longum everso succurrere seclo:

Terrarum \& curas gerere \& fulcire ruentes.

(Leo X. Pont. Max. 95-101),

genauso, wie in der Karl V. zugeeigneten:

Salve Heros, salve afflictis lux addita rebus

Magne Pater, per quem Romana potentia caelo

Attollet caput, \& famam super inferet astris.

(Carolus V. Imp. 59-61)

Die ehemalige Popularität von Zanchi zeigt sich darin, dass der Heidelberger Petrus Lotichius Secundus in seiner den Kurfürsten Otto-Heinrich preisenden Ekloge Nicer diese Zeilen fast wortwörtlich übernimmt:

Salve Heros, salve o nostris Sol addite terris,

Imperioque bonus rebusque labantibus adsis.

$(83-84)^{20}$

Öfter noch als die räumliche Entferntheit des idealen Herrschers wird die zeitliche Getrenntheit, die Gegenüberstellung der werteverlustigen Gegenwart und der augusteischen Zeit thematisiert. Selbstverständlich wurde diese Gegenüberstellung zu einem bis zum Überdruss wiederholten Gemeinplatz der humanistischen Dichtung. Wie Beccadelli in einem seiner Epigramme gallig anmerkt, mochte Augustus die Musen und die gelehrten Dichter deswegen, weil er selber ein Gelehrter war: Augustus Musas ac doctos doctus amavit. ${ }^{21}$ Die kunstvollsten Ausformulierungen dieses beinahe mechanisch wiederholten Loci communes finden sich allerdings bei den bukolischen Dichtern. Auch hier ist der Einfluss der Hirtendichtung des Calpurnius Siculus grundlegend. Calpurnius versucht nämlich nicht nur die bukolische Dichtung Vergils zu wiederholen, sondern geradezu die dichterische Laufbahn des vates sacer zu imitieren, durch die Planung nicht nur des Euvres, sondern auch der Biographie nach dem klassischen Vorbild, dessen Bedingung natürlich das Erringen der erhofften Unterstützung des Patrons ist:

Tu mihi talis eris, qualis qui dulce sonantem

Tityron e silvis dominam deduxit in urbem

ostenditque deos et ,, spreto “ dixit ,ovili

Tityre, rura prius, sed post cantabimus arma“.

(IV 160-163)

${ }^{20}$ Parnassus Palatinus. Humanistische Dichtung in Heidelberg und der alten Kurpfalz. Hrsg. von W. KÜHLMANN, H. WiEGAND. Heidelberg 1989, 56-60. Zanchis Wirkung wird bereits im Kommentar von Pieter Burman dem Jüngeren registriert: Petri Lotichii Secundi Solitariensis Poëmata Omnia... recensuit, notis et praefatione instruxit PETRUS BuRMANNUS SECUNDUS. Amstelaedami MDCCLIV, I 587593. Über die Rolle der Flussgötter in der zeitgenössischen panegyrischen Lyrik siehe DEUFERT, D.: Matthias Bergius (1536-1592). Antike Dichtungstradition im konfessionellen Zeitalter. Göttingen 2011, $247 \mathrm{ff}$.

\footnotetext{
${ }^{21}$ Epigr. VI (Pro Paride) 7.
} 
Eines der schönsten Beispiele dafür ist die fünfte Ekloge des Baptista Mantuanus ( $D e$ consuetudine divitum erga poetas). ${ }^{22}$ Der als Christianus Maro bekannte Dichter diente ganz bis zum 18. Jahrhundert als Schullektüre. Dass er für den zweiten Vergil gehalten wurde, hing wohl auch damit zusammen, dass er sein Leben im Karmelitenkloster in Mantua verbracht hat. Seine Eklogen stehen aber Juvenal viel näher als Vergil. ${ }^{23}$ Die fast mit naturalistischen Farben gezeichnete Landschaft bildet den krassen Gegensatz zur magischen Arkadien Vergils: Sie ist der Ort der harten Arbeit und des Elends, wo ungeschliffene Hirten und Bauern gleichermaßen um den Lebensunterhalt kämpfen. In der sechsten Ekloge (Cornix) wird das Hauptmotiv der ersten Ekloge Vergils und der siebten Ekloge des Calpurnius, der Erlebnisbericht des Stadtbesuchs eines Hirten, neu geschrieben. Während aber der Stadtbesuch des Hirten in den Eklogen von Vergil, Calpurnius und Nemesian zur Metapher der Dichterkarriere und des Herrscherlobs wurde, ${ }^{24}$ berichtet Cornix den in ihrer Winterunterkunft am Abend in Ruhe weilenden Hirten nicht über die Herrlichkeit von Rom, sondern über die Immoralität des städtischen Volkes. Die Stadt wird zum Grund allen Übels, zum Symbol des universalen Verfalls. Auf diese Weise wird der Topos des Besuchs in Rom auch in der fünften Ekloge in ihr Gegenteil gekehrt. Silvius fragt Candidus, den im zerrissenen Mantel vor Kälte zitternden Dichter (paenula ... trita, genu nudum, riget hispida barba, 22-23), etwas spöttisch, warum er denn nicht nach Rom fahre, um sein Glück zu versuchen, wo es doch so viele Poeten gibt und es so einfach ist, reich zu werden (ubi tot vates, ubi copia rerum, 111). Candidus antwortet verbittert, dass er Rom schon besucht hat, aber was hat es ihm genützt. Augustus ist schon längst tot und wird aus der Unterwelt nie zurückkehren. Damals waren es andere Zeiten, denn auch Tityrus hatte Wiesen, Tiere und Landstücke erhalten und von den Kriegen gesungen, denn die finanzielle Sicherheit verlieh ihm Beredsamkeit:

Tityrus - ut fama est - sub Maecenate vetusto rura, boves et agros et Martia bella canebat altius et magno pulsabat sidera cantu. Eloquium fortuna dabat; nos debile vulgus pannosos macie affectos, farragine pastos Aoniae fugiunt Musae, contemnit Apollo. [...]

Romana palatia vidi, sed quid Roma putas, mihi proderit? o Silvane, occidit Augustus numquam rediturus ab Orco.

(V 86-91; 119-121)

\footnotetext{
timore 1911

${ }^{22}$ The Eclogues of Baptista Mantuanus. Ed. with Introduction and Notes by W. P. MUSTARD. Bal-

${ }^{23}$ Über den satirischen Hintergrund der Eklogen des Mantuan siehe FETKENHEUER, K.: Hungernde Dichter, unwillige Mäzene. Baptista Mantuanus’ Ekloge V und die römische Satire. Philologus 156 (2012) 301-327.

${ }^{24}$ Siehe LuCK, G.: Besuch in Rom. In Festschrift für R. Muth. Hrsg. von P. HÄNDEL und W. MEID [Innsbrucker Beiträge zur Kulturwissenschaft]. Innsbruck 1983, 231-236; KÜPPERS, J.: Tityrus in Rom. Bemerkungen zu einem vergilischen Thema und seiner Rezeptionsgeschichte. ICS 15 (1989) 33-47.
} 
Die Erinnerung an Vergils Karriere wurde ein oft wiederkehrendes Motiv der satirischen Eklogen, und weil Mantuan den größten Einfluss auf die protestantischen Länder ausgeübt hatte, war es unter den Dichtern der deutschen Reformation besonders häufig vorzufinden. Auch der Aepolus der neunten Ekloge des Euricius Cordus (1486-1535) erinnert sich nostalgisch an den pius Augustus und den clarus Maecenas, die auch selber Dichter waren, und stellt dieses glückliche goldene Zeitalter seiner barbarischen Zeit gegenüber: Heute verlacht der Pöbel ohne Kunstsinn den Gesang der Hirten:

\section{(Aep.) Audio quod quidam Mantous Tityrus olim obtinuit blandis amissum cantibus agrum. \\ Si potes \& quid habes, Augustas carmen ad aures concine, compositas supplex meditare cicutas. Forsitan hae poterint aliquam tibi ferre salutem. (Lol.) Consulis in cassum: priscis id profuit annis: Cum pius Augustus cum clarus erat Moecaenas Carminum amatores nostrorum ipsique Poetae. Barbara iam currunt contemptis saecula Musis.}

$$
(\text { Aegl. IX 118-122) })^{25}
$$

Ein Zeitgenosse von Mantuan war Fausto Andrelini, der, wie Erasmus schreibt, diu regnavit Lutetiae, und der den auch Anlass zum Scherz gebenden Titel poeta regius ac regineus, poète du roi et de la reine gewann. ${ }^{26}$ Andrelini konnte sich nicht beschweren, denn auch Vergil selbst hätte ihn für seine hohe Leibrente beneidet. Allerdings erscheint dieses Motiv auch bei ihm: Hätte Augustus Vergil nicht unterstützt, wäre er nie zur epischen Dichtung gelangt, sondern nur bis zur Verfassung des Culex:

Sublatus ab ipso

Tityrus Augusto Mavortia coeperat arma horribili resonare tuba, pressusque maligna paupertate miser nullaque afflatus ab aura vix nimium rudibus culicem deflevit avenis.

Ein konstanter Bestandteil dieser Textstellen - seit Calpurnius Siculus und Nemesianus - ist das Bestreben des Dichters, sich von der Bukolik zu verabschieden und höheren Gattungen zuzuwenden, denn sie wollten nicht nur mit der Hirtendichtung Vergils wetteifern, sondern die dichterische Laufbahn des vates sacer nachahmen. Die Abwesenheit oder der adventus des Herrschers, das Fehlen oder das Vorhandensein seiner Unterstützung ist also zugleich auch die Bedingung für den Fortschritt in der Hierarchie der Gattungen. In der Hieron II. gewidmeten Idylle des Theokritos

${ }^{25}$ Humanistische Lyrik des 16. Jahrhunderts (Anm. 18) 236-244.

${ }^{26}$ The Eclogues of Faustus Andrelinus and Ioannes Arnolletus. Ed. with an Introduction and Notes by W. P. MuSTARD. Baltimore 1918. Über die Karriere des Andrelini siehe TOURNOY-ThOEN, G.: Fausto Andrelini et la cour de France. In L'humanisme français au début de la Renaissance. Paris 1973, 65-79.

${ }^{27} \mathrm{Vgl}$. Martialis 8. 55. 19-20: Protinus Italiam concepit et arma virumque, / qui modo vix culicem fleverat ore rudi. 
(XVI, Charites) hat der Dichter die Aufeinander-Angewiesenheit des der werteverlustigen Gegenwart nur schmachtenden Dichters und des zur Wiederherstellung des entschwundenen goldenen Zeitalters berufenen Herrschers mit einer typisch homerischen Wendung voller prophetischer Obertöne formuliert: Es wird der Mann kommen, der

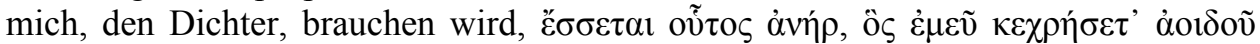
(73). Das Hirtengedicht soll vor allem beweisen, dass der junge Dichter bereit ist, seine Begabung auch in höheren Gattungen auf die Probe zu stellen, und dass er, wenn er entsprechend unterstützt wird, in der Lage ist, die neue goldene Zeit würdig $\mathrm{zu}$ verewigen.

Um ein spätes Beispiel zu nennen: In seinem Hirtenroman Coridon und Phyllis beschreibt der schlesische Daniel von Czepko (1605-1660) ganz offen den dichterischen Karrierismus:

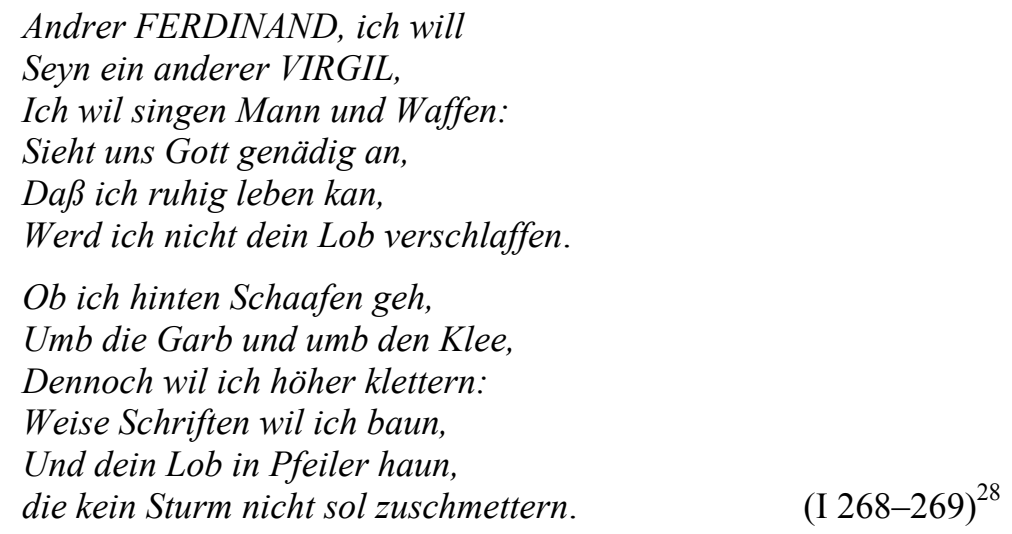

Ob ich hinten Schaafen geh,

Umb die Garb und umb den Klee,

Dennoch wil ich höher klettern:

Weise Schriften wil ich baun,

Und dein Lob in Pfeiler haun, die kein Sturm nicht sol zuschmettern.

Dies führt jedoch zur stetigen Übertretung der von der rota Vergili vorgeschriebenen, die drei Stilebenen trennenden Gattungshierarchie: Die dem genus humile angehörende Gattung interferiert mit dem genus grande. Das beste Beispiel für den entscheidenden Charakter des epischen Stils liefern vielleicht die politischen Eklogen Petrarchs: Reminiszenzen aus dem Aeneis, von Lucan, Statius und Claudian sind viel häufiger als die aus Vergils Eklogen. ${ }^{29}$ Als Cola di Rienzo in der fünften Ekloge als

${ }^{28}$ Daniel von Czepko: Sämtliche Werke. Hrsg. von H-G. ROLOFF und M. SzYROCKI. Berlin 1988.

${ }^{29}$ Bezeichnenderweise stammt die überwiegende Mehrheit der vergilischen Reminiszenzen nicht einmal aus den Eklogen, sondern aus dem Aeneis, mehr noch, die Bilder der Hirtenwelt entstammen in zahlreichen Fällen auch nicht der bukolischen, sondern der epischen Dichtung: saturata... armenta (Argus 4-5) kommt nur in der Geschichte des Cacus vor (Aen. VIII 213-214), pastorum turba (Argus 20) ruft ein Vergleich aus der Thebais des Statius (VIII 691-692) wach, während die nächste Parallele zu luctus Driadum (Argus 89) bei Claudianus (De raptu 381) vorliegt. Es ist also irreführend, bezüglich des Bucolicum carmen von ,vergilischen“ Eklogen zu reden, wie das in der Fachliteratur bis heute - nicht selten - der Fall ist, siehe z.B. den Aufsatz von A. BUCK: Die antiken Autoren aus der Sicht Dantes und Petrarcas. In Die Rezeption der Antike. Zum Problem der Kontinuität zwischen Mittelalter und Renaissance. Vorträge gehalten anlässlich des ersten Kongresses des Wolfenbütteler Arbeitskreises für Renaissanceforschung in der Herzog August Bibliothek Wolfenbüttel vom 2. bis 5. September 1978. Hrsg. von A. BUCK [Wolfenbütteler Abhandlungen zur Renaissanceforschung 1]. Hamburg 1981, 14. 
der Retter von Rom und der Wiederhersteller seiner alten Macht erscheint, erschreckt er mit seiner Stimme die Völker von Mauretanien bis zu Indien:

Audit iam litus utrumque

Carmen et extremus calaber ligurumque recessus,

Undifragi sectique tenens convexa Pelori.

Si vocem extulerit, Mauros turbabit et Indos,

Arctoasque nives, austrique calentis arenas.

Die Allusion spielt eindeutig auf die Prophezeiung über Augustus im sechsten Buch des Aeneis an:

super et Garamantas et Indos

proferet imperium; iacet extra sidera tellus,

extra anni solisque vias, ubi caelifer Atlas

axem umero torquet stellis ardentibus aptum.

huius in adventum iam nunc et Caspia regna

responsis horrent divum et Maeotia tellus, et septemgemini turban trepida ostia Nili.

Diesbezüglich hat dann Boccaccio seine kaum kaschierte Missbilligung zum Ausdruck gebracht: In seinem an Fra Martino da Signa geschriebenen Brief merkt er an, dass sein Meister einen ungewöhnlich erhabenen Ton angeschlagen hat: stilum preter solitum paululum sublimavit. Doch gibt er zu, dass Vergils Eklogen einen versteckten Sinn haben: sub cortice nonnullos abscondit sensus. ${ }^{3}$

Gerade auf dieser Grundlage wird in späteren Poetiken die gehobenere Spielart der bukolischen Dichtung legitimiert. Wie Cristoforo Landino (1424-1498) in seinem Vergil-Kommentar schreibt: Obwohl dieser zu göttlichen Sachen geborene Dichter sich in seinem jüngeren Alter in der niedrigeren Stilebene geübt hat, hat er bereits hier Erhabenes geschaffen:

Hoc tamen in Vergilio admiror [...] ut quamvis a persona pastorali non discedat, tamen alium sensum longe excellentiorem sub illo vulgari abscondit [...]. Et profecto ad maxima quaeque ac divina natus hic poeta,

${ }^{30}$ Der neueste, mit wissenschaftlichem Anspruch fertiggestellte Kommentar des Bucolicum carmen ist Pétrarque, Bucolicum carmen. Texte latin, traduction et commentaire par M. FRANÇOIS et P. BACHMANN. Avec la collaboration de F. RoudAut. Préface de J. MEYERS. Paris 2001. Über die Ekloge siehe neulich FENZI, E.: Per Petrarca politico: Cola di Rienzo e la questione romana in „Bucolicum Carmen V, Pietas Pastoralis“. Bollettino di italianistica 1 (2011) 49-88.

${ }^{31}$ Post hunc autem scripserunt et alii, sed ignobiles, de quibus nil curandum est, excepto inclito preceptore meo Francisco Petrarca, qui stilum preter solitum paululum sublimavit et secundum eglogarum suarum materias continue collocutorum nomina aliquid significantia posuit. Ex his ego Virgilium secutus sum, quapropter non curavi in omnibus colloquentium nominibus sensum abscondere; et ob id, cum desideres tam titulorum quam etiam nominum colloquentium in eglogis meis sensum, nolo mireris, magister optime, si absque significato nonnulla colloquentium nomina comperies: de titulis non sic, omnes enim accurate apposui. Ep. 23. 
ita in humilioribus a prima aetate se exercuit, ut iam tum maiora illa mente conceperit. ${ }^{32}$

In seinem Werk De poeta schreibt Antonio Minturno (1500-1577), dass Vergil als Erster gezeigt hat, wie man der Gattung scheinbar fremde Themen richtig bearbeiten kann:

Mihi vero ille vel hoc praecipue mirabilis videtur, quod cum agrestes humilesque personas, quae nihil non rusticum simplexque loquantur, introducat, lusu illo gravia quaedam abdite comprehendit, opusque per se nudum ac tenue sic exornat, ut cum exteriorem habitum, et personae convenientem nunquam exuat, interiorem induat politiorem in modum, atque cum in id quod apparet, incumbat, id quod latet, probe conficiat. [...] Is poetarum sane mos est, ut fictis vera occulte, ludo seria complectantur. Nec vero in tribus illis Eclogis, quas minus esse Bucolicas arbitrantur, pastoralem personam abiecit. [...] Itaque primus docuit quae a Bucolica ratione putantur abhorrere, quam probe attingi possint. ${ }^{33}$

Deswegen halten wir die psychologisierende Annäherungsweise des Erforschers der Gattung, S. Chaudhuri, für kritikwürdig, der nach dem Muster des bekannten Terminus anxiety of influence von H. Bloom den Grund für die Experimentierung mit dem epischen Stil in der Angst vor Bedeutungslosigkeit (anxiety of insignicance) zu finden meint. ${ }^{34}$ Ganz im Gegenteil: Die bukolische Gattung ist berufen, als eine Art Vorübung zu beweisen, dass der junge Dichter bereit ist, seine Begabung auch in höheren Gattungen auf die Probe zu stellen. ${ }^{35}$ Zudem darf man nicht aus den Augen verlieren, dass die panegyrischen Eklogen in den oft vierzehn, sechszehn oder mehr Stücke umfassenden Sammlungen keineswegs überwiegen. Andererseits hat bereits G. Ellinger darauf hingewiesen, dass diese panegyrischen Gedichte für die zeitgenössischen Leser

${ }^{32}$ Zitiert von CHAUDHURI, S.: Paulo maiora canamus: The Transcendence of Pastoral in the NeoLatin Eclogue. CRCL 33 (2006) 91.

${ }_{33}$ Antoni Sebastiani Minturni De poeta, ad Hectorem Pignatellum, Vibonensium ducem, libri sex. Venetiis, ann. MDLIX. 163

${ }^{34}, \ldots$ an anxiety of insignificance troubling pastoral poets in a dispensation geared to the epic. Even while writing eclogues, poets generally view the form against the full hierarchy of genres, and work into it the substance of other modes and forms - even those specifically not-pastoral, even those which the pastoral is definitively avoiding or opposing." a. a. O. 98. Einigermaßen erinnert das an den Begriff „anxiety of originality“ von GREEN, TH.: The Light in Troy. Imitation and Discovery in Renaissance Poetry. Yale University Press 1982, 195. In der Monographie von TH. HuBBARD begegnet der Ausdruck „anxiety of posterity": „We see, moreover, a heightened anxiety not only about the author's relation to the past, but about his relation to posterity as interpreted through the framework of the past." The Pipes of Pan. Intertextuality and Literary Filiation in the Pastoral Tradition from Theocritus to Milton. Ann Arbor $1998,248$.

35 „As much as the Renaissance admired pastoral poetry, as much as it praised its naturalness and simplicity, it was at the same time aware that the aspiring poet, like the great men celebrated in and by pastoral, deserved and required a higher style, a greater argument. Pastoral is, then, repeatedly aiming at something greater, at ascending the scale of generic progression." Cullen, P.: Spenser, Marvell and Renaissance Pastoral. Cambridge 1970, 10. 
eine ganz andere Bedeutung hatten. ${ }^{36}$ Nach der treffenden Definition von K. Garber bilden die biographische Allegorie sowie die Aufgeschlossenheit für Aktualitäten, d.h. der Biographismus und der Feuilletonismus, zusammen mit dem Panegyrikus die drei konstitutiven Merkmale der humanistischen Bukolik. ${ }^{37}$

Andererseits haben die späteren Dichter, im Gegensatz zu dem von vornherein im gehobenen Stil schaffenden Petrarch, den im höheren Stil geschriebenen Text scharf abgetrennt, entweder mit der Entdeckung einer in einen Baum oder einen Felsen geritzten göttlichen Prophezeiung oder mit der Erzählung von Silenus, Proteus oder einer Flussgottheit. Die Rahmengeschichte haben sie jedoch, wie schon in Piccolominis Ekloge zu beobachten war, so bukolisch wie möglich gestaltet. Ein musterhaftes Beispiel für die scharfe Absonderung des genus humile und des genus grande war die bereits erwähnte erste Ekloge des Calpurnius: Als die Hirten die in den Baum geritzte Prophezeiung finden, ruft Ornytus aus:

Non pastor, non haec triviali more viator, sed deus ipse canit: nihil armentale resultat, nec montana sacros distinguunt iubila versus.

Die Hirten treten also nicht als Dichter, sondern als Leser eines im höheren Stil geschriebenen Textes auf. Die Einbettung des Panegyrikus in einen fiktiven Rahmen spielt eine doppelte Rolle: Die Geschichte scheint zwar von der unbedingten Bewunderung des Herrschers im Kreise des einfachen Volkes zu zeugen. Der Effekt der Schmeichelworte wird aber dadurch beeinträchtigt, dass ihre Unmittelbarkeit aufgehoben wird, indem sie ironisch distanziert dargestellten Figuren in den Mund gelegt werden. Um nur eine unter den unzähligen Imitationen zu nennen: In der bereits erwähnten Ekloge des Lotichius Secundus sind es die Musen selber, die die Gesänge in die Stämme der Kirschbäume ritzen - und für den epischen Stil ließe sich kaum ein besseres Beispiel finden als die darauf folgende Aeneis-Paraphrase:

Et vos o gelidae nemorum gaudebitis umbrae,

Ludentes ubi mane mane canent, ubi vespere Musae,

Et mansura sui longum monumenta per aevum

Dulcia purpureis incident carmina truncis.

Innumeris alii se tollunt laudibus amnes,

${ }^{36}$ „Um den richtigen Maßstab zur Beurteilung dieser idyllischen Dichtung zu gewinnen, muß man sich auf den Standpunkt der Zeitgenossen zu stellen suchen. Die den Eklogen zugrunde liegenden Ereignisse und Tatsachen waren das Gespräch des Tages und erfüllten alle Gemüter; man kann daher wohl annehmen, daß trotz der maskierten Darstellung der Inhalt jedem sofort gegenwärtig war und das Verständnis nirgends auf Schwierigkeiten stieß. Für den heutigen Leser hat die Verwendung der pastoralen Form freilich viel Befremdliches; der Wiederspruch zwischen dem Inhalt und der gewählten Form macht sich oft so stark geltend, daß von einer ersten Wirkung nicht die Rede sein kann.“ ELLINGER (Anm. 12) II 89.

${ }^{37} \mathrm{Der}$ locus amoenus und der locus terribilis. Bild und Funktion der Natur in der deutschen Schäfer- und Landlebendichtung des 17. Jahrhunderts. Köln-Wien 1974, 13.

${ }^{38}$ Über die erste Ekloge des Calpurnius siehe neulich EsPOSITO, P.: La profezia di Fauno nella I ecloga di Calpurnio Siculo. In Fer propius tua lumina. Giochi intertestuali nella poesia di Calpurnio Siculo. Incontri sulla poesia latina di età imperiale. A cura di L. LANDOLFI, R. ODDO. Bologna 2009, 13-39. 
credo equidem: varios sub gurgite pisces

Rhenus alit, fontesque suos, suaque ostia iactat

Danubius, genus acre virum pater educat Albis,

magnanimos Nicer Heroas rerumque potentes,

insignes pietate Duces et fortibus ausis.

Der epische Stil bedeutet aber auch, dass das in den panegyrischen Eklogen erscheinende goldene Zeitalter sich von der im religiösen Sinne verstandenen goldenen Zeit der vierten Ekloge Vergils scharf trennt. Bei Calpurnius bedeutet die goldene Zeit nicht mehr den Eintritt in ein sich nach kosmischen Gesetzen richtendes Weltzeitalter, sondern bloß die (Wieder)Herstellung der Rechtsordnung, die Sicherheit und Friede gewährende Herrschaft des neuen Prinzeps. Anstelle der mythischen Urzeit der Menschheit übernimmt also die Herrschaft des Augustus die Rolle der vergangenen goldenen Zeit. Diese Betrachtungsweise war offenbar besser adaptierbar für den Lob von Herrschern verschiedenen Ranges als die vierte Ekloge Vergils. Demnach ist H. Levin kaum ohne Vorbehalte zuzustimmen, wenn er sagt, dass diese gewissermaßen eigenständige Unterart (minor genre) der Bukolik unter dem Einfluss der vierten Ekloge Vergils zustande kam, welche den herrscherlobenden Dichtern als ständiges Vorbild diente. $^{39}$

Wie die Prophezeiung des Faunus in der ersten Ekloge des Calpurnius die Paraphrase der Wahrsagung im ersten Buch des Aeneis darstellt, so befolgen auch die Prophezeiungen bei den neulateinischen Dichtern das epische Schema.

In der neunten Ekloge des Florentiner Naldo Naldi (1436-1513) tritt Silvanus als der sachkundige Arzt der Krankheiten der Herde auf, woraus sich leicht ermitteln lässt, dass er mit der Person des alten Cosimo de' Medici identifizierbar ist. Seine Lehre behandelt die Kunst des Herrschens in der Sprache der bukolischen Symbolik. Beachtenswert ist allerdings, dass auch Cosimo sich vom wirklichen, mythologischen goldenen Zeitalter abgrenzt: Er ist ein guter Arzt seines Volkes (quin etiam medicas trado, quis crederet, artes, IX 65), aber mit Nektar strömende Flüsse und Honig tröpfelnde Bäume kann nicht einmal er nach Florenz zaubern:

Nullus enim nostrae subit haec oracula, mentis

qui non ediscat dirum sedare furorem, atque odiis posuisse modum. Discordia longe

tristis abest; nullis fertur violentia saeptis, tutus ab insultu pastor sua quisque tuetur pascua, militibus non diripienda malignis. me duce Gradivus durissima vincula sensit, ne queat Etruscos Furiis vexare colonos. otia sic dempto peraguntur grata labore, omnibus et pleno colitur pax candida cornu. securae interea tondent virgulta capellae, ulla nec insidias metuunt armenta luporum,

${ }^{39}$ The Myth of Golden Age in the Renaissance. Bloomington-London 1969, 18. 
ut nihil haec aetas intersit et aurea quondam, haec nisi quod fluvios non vidit nectare plenos currere, nec quercus emittere roscida mella.

$(\text { IX } 70-82)^{40}$

Ein immer wiederkehrendes Motiv solcher epischen Darstellungen der goldenen Zeit ist - im Gegensatz zu den antiken Beschreibungen -, dass die Schifffahrt nicht abgebrochen wird, sondern, im Gegenteil, die Meere sicher werden, das Dankgebet der alexandrinischen Kaufleute aus Suetons Biographie des Augustus gleichsam wachrufend: per illum se vivere, per illum navigare, libertate atque fortunis per illum frui. ${ }^{41}$ Ein schönes Beispiel dafür liefert bereits Piccolominis Ekloge:

Tuta mari navis totoque sub orbe viator tutus erit nullique legent achonita, sed ipsa quique data felix contentus sorte quiescit.

Dasselbe Motiv findet sich bei Giambattista Amalteo in seiner die Macht des venezianischen Patrizius Federico Savorgnan verherrlichenden Ekloge Silis:

Te duce, casta Fides cum fecerit otia terris,

Aurea restituent concordes secula Parcae:

Et praeceps toto fugiet Discordia mundo.

[...]

Tunc impune lupis audax miscebitur agna:

Cyaneas etiam cautes, Scyllaeaque saxa

Transmittent tuto volitantia carbasa ponto,

Nec pelago abruptas horrebit nauta procellas.

$(70-72 ; 76-79)$

Ähnlicher Weise beschreibt Cornelio Amalteo das nach dem Sieg von Lepanto eintretende goldene Zeitalter in seiner oben erwähnten Ekloge:

Ilice praeterea stillabunt dulcia mella,

Et pecudes multo distendent ubera lacte,

Colludentque lupis medijs in vallibus agnae,

Quinetiam placidis sternentur fluctibus aequor,

Nec Scyllam metuet pinus, nec nauta Charybdim,

Spirabunt Zephyri tantum, quorum omnia flatu

Ridebunt, totusque adeo laetabitur orbis.

(Proteus 75-81)

Dem ist auch dann so, wenn das Gedicht selbst dem Schema der vierten Ekloge Vergils folgt: Das Gedicht der größten Gestalt des schottischen Humanismus, George Buchanan, feiert die Geburt des Königs Jakob VI. (Genethliacon Jacobi Sexti Regis

${ }^{40}$ Naldi Naldii Florentini Bucolica Volaterrais Hastiludium Carmina Varia. Ed. W. L. GRANT. Florentiae MCMLXXIV. Anzumerken ist, dass Naldi elf Eklogen verfasst hat, offensichtlich nach dem Muster der gemeinsam tradierten Sammlung von Calpurnius und Nemesianus. Über die politischen Bezüge der Eklogen von Naldi siehe neulich MARTELLI, M.: Letteratura fiorentina del Quattrocento. Il filtro degli anni sessanta. Firenze 1996, $79 \mathrm{ff}$.

${ }^{41}$ Aug. 98. 2. 
Scotorum): Die Darstellung der goldenen Zeit selbst entbehrt jegliches mystische Element und hält sich an die epischen Motive, sie prophezeit nur das Zu-Ende-Kommen der Feindseligkeit zwischen Angelsachsen und Schotten und die Segen des Friedens - diese Beschreibung wurde von Buchanans Monographisten mit Recht als „political paradise" bezeichnet: ${ }^{42}$

Cresce, puer, patriae auspiciis felicibus orte, Exspectate puer, cui vatum oracula priorum Aurea compositis promittunt saecula bellis. Tuque peregrinis toties pulsata procellis Pene tuo toties excissa Britannia ferro Exsere laeta caput, cohibe pacalis olivae Fronde comam, repara flammis foedata, ruinis Convulsa, \& pulso cole squalida tecta colono. Pone metum, aeternam spondent tibi sidera pacem. Iam neque Saxonidae Scotos, nec Saxona Scotus Infestus premet, \& cognato sanguine ferrum Polluet, \& miseras praedendo exhauriet urbes.

(Silvae VII 15-26) ${ }^{43}$

Anderswo lässt sich beobachten, dass auch die aus Vergils vierten Ekloge übernommenen Motive sich ans epische Schema passen. Die vierte Ekloge aus der Sammlung Piscatoriae von Nicola Giannettasio (1648-1715) feiert die Siege des deutsch-römischen Kaisers und ungarischen Königs Leopold I., die Abwehr der türkischen Belagerung von Wien und die Rückeroberung des ungarischen Erzbischofssitzes, Esztergom (Gran). Die centoartig zusammengewebten vergilischen Verse erhalten einen absolut neuen Sinn: Mit Hilfe der Heiligen Mutter Gottes befreit Leopold die ganze Erdkugel von der Angst, und auch wenn Spuren der früheren Sünden noch vorzufinden sind, werden auch diese langsam vergehen, und auf der ganzen Welt wird der wahre Glaube walten: Das bei Vergil erscheinende wundervolle Kind verwandelt sich in den von Gott geliebten Helden des Glaubens:

Casta fave nunc Virgo; tuo si numine CAESAR

Vicit, \& ingenti terras formidine solvit,

Hoc duce, si qua manent priscae vestigia culpae,

Tollentur, totoque Fides regnabit in orbe.

Gens sancta ex alto mittetur, \& impia passim

Desinet: eque polo veniens Astraea redibit

${ }^{42}$,It is a political paradise which he is foretelling, in which the only beasts that will lie down together in peace are the lion and the unicorn of England and Scotland." FORD, PH. J.: George Buchanan, Prince of Poets. With an Edition of the Miscellaneorum liber. Aberdeen 1982, 105.

${ }^{43}$ Georgii Buchanani, Scoti, Poëtarum sui seculi facile principis Opera Omnia. Lugduni Batavorum MDCCXXV. 
In terras iterum, \& Saturnia secla reducet;

Pacatumque reget totum sub CAESARE Mundum. $\quad(12-19)^{44}$

Bis zur Zeit der Renaissance werden die Legenden um die im Mittelalter durchaus verbreitete sog. Augustustheologie, so die wundersamen Geschichten der Sibylla Tiburtina und der Errichtung des Ara Caeli, ziemlich in den Hintergrund gedrängt. ${ }^{45}$ Schon im Epos De partu Virginis des Sannazaro steht so viel, dass Augustus für die Zeit der Geburt des Erlösers den universalen Frieden mitgebracht hat:

Interea terra parta iam pace marique

Augustus pater aeratis bella impia portis

clauserat et validis arctarat vincta catenis;

dumque suas regnator opes viresque potentis

imperii exhaustasque armis civilibus urbes

nosse cupit, magnum censeri iusserat urbem ... $\quad(116-121)^{46}$

Das Motiv des nach siegreichen Kriegen eintretenden universalen Friedens ist jedoch vielleicht das konstanteste Motiv der die augusteische Zeit heraufbeschwörenden Gedichte. ${ }^{47}$ Es erscheint, gleichsam formelhaft, bereits bei Giovanni Marrasio (1400/1404-1452) in seinem den ungarischen König Sigismund lobenden Gedicht:

Maecenas studiis cum sis et Iulius armis, Augustus pacis totius orbis eris. $\quad$ (Carm. 20. 13-14) ${ }^{48}$

Aus diesem kurzen Überblick ist vielleicht klar geworden, dass das Herrscherbild der neulateinischen Bukolik trotz der bedeutenden zeitlichen und räumlichen Entfernungen überraschend einheitlich ist. Auch bei voneinander vollkommen unabhängig schaffenden Dichtern erscheint die Grundsituation der achten Ekloge Vergils immer

${ }^{44}$ Nicolai Parthenii Giannettasii Neapolitani e Societate Jesu Opera Omnia Poëtica. Neapoli MDCCXV. Über die Fischer-Eklogen siehe SCHINDLER, C.: Vitreas Crateris ad undas. Le egloghe piscatorie di Nicolò Partenio Giannettasio (1648-1715). StudUmanistPicen 23 (2003) 293-304.

${ }^{45}$ Siehe dazu Frauenholz, E. V.: Imperator Octavianus Augustus in der Geschichte und Sage des Mittelalters. Historisches Jahrbuch 46 (1926) 86-122; OPELT, I.: Augustustheologie und Augustustypologie. JbAC 4 (1961) 44-57; BuRKE, P. F.: Augustus and Christianity in Myth and Legend. NECJ 32 (2005) 213-220.

${ }^{46}$ Über den Einfluss der vierten Ekloge Vergils auf Sannazaros Meisterwerk siehe BINDER, G.: Goldene Zeiten: Immer wieder wird ein Messias geboren. Beispiele neuzeitlicher Aneignung der 4. Ekloge Vergils. In Vestigia Vergiliana. Vergil-Rezeption in der Neuzeit. Hrsg. von TH. BURKARD, M. SCHAUER, C. WIENER [Göttinger Forum für Altertumswissenschaft - Beihefte Band 3 Neue Folge]. Berlin 2010, $52-57$.

${ }^{47}$ Der Befund von U. Töns über Sannazaro besitzt auch allgemeine Gültigkeit: „Bukolik wird hier zum Symbol einer politischen Utopie, die mit Caesar und Augustus vor der Verwirklichung steht. In den Eklogen ist - sehr zurückhaltend allerdings - angedeutet, was Vergil in der Aeneis als Ideologie des augusteischen Friedensreiches deutlicher verkündet. Schließlich sollte man bedenken, daß auch die georgischen Züge, die sich in den Eklogen finden, in Vergils Denken einen Zusammenhang mit der Goldenen Zeit Saturns besitzen." TÖNS, U.: Sannazaros Arkadia. Wirkung und Wandlung der vergilischen Ekloge. $A \& A 23$ (1977) 154 .

${ }^{48}$ ReStA, G.: I. Marrasius: Angelinetum et Carmina varia. Palermo 1976. 
wider, das Thema der Entferntheit bzw. des adventus des Herrschers, und der SilenusGesang der auch oft nachgeahmten sechsten Ekloge wird - teilweise oder ganz - immer wieder durch die epische, die Aeneis imitierende Prophezeiung der ersten Ekloge des Calpurnius ersetzt. Auch wenn ab und zu einzelne Motive aus Vergils vierten Ekloge übernommen werden, unterscheidet sich die in diesen Werken dargestellte goldene Zeit entschieden von Vergils Prophezeiung über den kosmischen Weltzeitalterwechsel, der den allmählichen Rückgang der Zivilisation und die Entfaltung der paradiesischen Zustände mit sich bringt. Eine Rolle dabei kann die Tatsache gespielt haben, dass die eschatologische Interpretation des vergilischen Werks sich zäh gehalten hat: Von den auffallenden Entsprechungen zwischen der vierten Ekloge und dem Jesajabuch inspiriert veröffentlichte Alexander Pope 1713 ein Gedicht in der Zeitschrift Spectator (Messiah, A Sacred Eclogue), das Motive von Vergil bzw. aus dem Jesajabuch verschmilzt und die Textparallele in den beigefügten Notizen auch gründ-

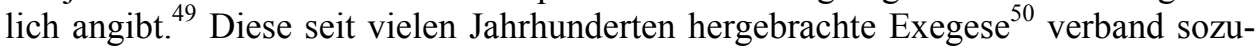
sagen die vierte Ekloge mit den heiligen Texten, wodurch das darin nachgezeichnete Bild der goldenen Zeit sich auch stark vom politisch geladenen, in der Hirtendichtung so oft dargestellten goldenen Zeitalter abhieb. Eine seltene Ausnahme bildet Bernard Le Bovier de Fontenelle, der im Gegensatz zu der Mehrheit seiner Zeitgenossen das Gedicht lediglich als ein die Geburt des Sohnes von Pollio feierndes und dem Patron zugleich schmeichelndes genethliacon betrachtete. Er formuliert jedoch seine Kritik genau auf dieser Grundlage. Seiner Ansicht nach ist die Anhäufung von märchenhaften und schleierhaften Phänomenen (toutes ces merveilles incompréhensibles) gänzlich verfehlt, und der Lob des Pollio wäre wirkungsvoller gewesen, wenn er die als Segen des Friedens eintretende goldene Zeit mit etwas wirklichkeitsnäheren Bildern nachgezeichnet hätte, das Glück des Landlebens selbstverständlich in den Mittelpunkt stellend. ${ }^{51}$ Das entspricht, wie wir gesehen haben, vollkommen der Darstellung der goldenen Zeit in der neulateinischen Bukolik, deren Vorbild die idealisierte Zeit der Herrschaft des Augustus bildete. ${ }^{52}$ Die epischen, die politische goldene Zeit vergegenwärtigenden Beschreibungen sind aber nicht nur berufen, die Größe des friedens-

${ }^{49}$ Von den Umständen der Entstehung der Ekloge und von ihrer Aufnahme liefert die Monographie von N. MURRAY GoldSMith ein ausgezeichnetes Bild: Alexander Pope. The Evolution of a Poet. Burlington 2002, $102 \mathrm{ff}$.

${ }_{50}$ Siehe neulich HEIL, A.: Christliche Deutungen der Eklogen Vergils. $A \& A 53$ (2007) 100-103.

51 ,Je ne sais cependant s'il ne devoit pas s'en tenir aux Muses pastorales; il eût fait une peinture agréable des biens que le retour de la paix alloit produire à la campagne, et cela, ce me semble, eût bien valu toutes ces merveilles incompréhensibles qu'il emprunte de la Sibylle de Cumes... [...] On auroit miex flatté Pollion par des chose qui eussent eu un peu plus de vraisemblance." LE BOVIER DE FONTENELle, B.: Les poésies Pastorales avec un Traité sur la Nature de l'Églogue et une Digression sur les Anciens et les Modernes. A la Haye, chez Antoine van Dole, MDCCXXXVI, 126.

${ }^{52}$ Vgl. die Bemerkung von K. GARBER: „Arkadische und (gelegenheitlich) georgische Elemente werden sich mit den genuin-episch-nationalen in der Frühen Neuzeit immer wieder dort kreuzen, wo es um die Universalisierung politischer Ansprüche im Namen umfassender Pazifizierung gehen wird..." Zur Konstitution der europäischen Nationalliteraturen. In Nation und Literatur im Europa der Frühen Neuzeit. Akten des I. Internationalen Osnabrücker Kongresses zur Kulturgeschichte der Frühen Neuzeit. Hrsg. von K. GARBER. Tübingen 1989, 10. 
schaffenden Herrschers, sondern auch die Fähigkeiten des zur Verewigung seiner Taten bereiten Dichters nachzuweisen, denn sie erhofften sich vom Eintritt des an die augusteische Zeit erinnernden goldenen Zeitalters den mit Vergils vergleichbaren Aufschwung ihrer eigenen Karriere.

Lajos Zoltán Simon

Lehrstuhl für Latinistik

Eötvös-Loránd-Universität (ELTE)

Budapest

Ungarn 